\title{
ANÁLISE DE VÍDEOS DO YOUTUBE QUE ABORDAM A TÉCNICA DE CATETERISMO URINÁRIO DE DEMORA FEMININO
}

Anna Patrícia Cavalcante de Morais Pinto', Marta Silvanêre Pereira Dantas², Pétala Tuani Candido de Oliveira Salvador ${ }^{3}$, Cláudia Cristiane Filgueira Martins Rodrigues ${ }^{3}$, Viviane Euzébia Pereira Santos ${ }^{4}$

'Discente de Enfermagem. Universidade Federal do Rio Grande do Norte. Natal-RN-Brasil.

${ }^{2}$ Enfermeira. Universidade Federal do Rio Grande do Norte. Natal-RN-Brasil.

${ }^{3}$ Enfermeira. Doutoranda em Enfermagem. Professora da Universidade Federal do Rio Grande do Norte. Natal-RN-Brasil.

${ }^{4}$ Enfermeira. Doutora em Enfermagem. Professora da Universidade Federal do Rio Grande do Norte. Natal-RN-Brasil.

RESUMO: Trata-se de uma pesquisa descritiva, de abordagem quantitativa, realizada no sítio de compartilhamento YouTube, cujo objetivo foi caracterizar os vídeos do YouTube que abordam a técnica de cateterismo urinário de demora feminino. A busca dos vídeos foi realizada em setembro de 2014 utilizando-se o descritor controlado "cateterismo urinário". Foram analisados 29 vídeos, dos quais apenas três estavam de acordo com os padrões estabelecidos pela literatura; dentre os principais erros encontrados, destacam-se a ausência de registro no prontuário, da lavagem das mãos e a utilização da mesma luva para a antissepsia e inserção do cateter. Apesar de o sítio de compartilhamentos de vídeo YouTube ser uma ferramenta amplamente difundida atualmente, há uma carência de vídeos que reproduzam a técnica de acordo com o que é preconizado na literatura, o que pode influenciar negativamente a população que o utiliza para fixar o conhecimento.

DESCRITORES: Cateterismo urinário; Enfermagem; Webcasts; Tecnologia educacional.

\section{ANALYSIS OF YOUTUBE VIDEOS ADDRESSING THE INDWELLING URINARY CATHETERIZATION PROCEDURE IN WOMEN}

\begin{abstract}
This is a descriptive research with a quantitative approach, carried out on the website YouTube to characterize YouTube videos addressing the indwelling urinary catheterization procedure in women. A search of the videos was carried out in September 2014 using the descriptor "urinary catheterization". Twenty-nine videos were analyzed, only three of which were in accordance with the standards established in the literature; among the main errors found, the absence of registration in the medical records, hand washing and use of the same glove for antisepsis and catheter insertion are highlighted. Even though YouTube is currently a widespread tool, there is a lack of videos reproducing the procedure according to literature recommendation, which can negatively influence the population that uses it to correct their knowledge.
\end{abstract}

DESCRIPTORS: Urinary catheterization; Nursing; Webcasts; Educational technology.

\section{ANÁLISIS DE VIDEOS DE YOUTUBE CON LA TEMÁTICA TÉCNICA DE CATETERISMO URINARIO DE DEMORA FEMENINO}

RESUMEN: Investigación descriptiva, de abordaje cuantitativo, realizada en el sitio de compartir YouTube, cuyo objetivo fue caracterizar los videos de YouTube que abordan la técnica de cateterismo urinario de demora femenino. La búsqueda de los videos fue realizada en septiembre de 2014 utilizándose el descriptor "cateterismo urinario". Fueron analizados 29 videos, de los cuales solamente tres estaban en los patrones establecidos por la literatura; entre los principales errores encontrados están la ausencia de registro en el prontuario, no lavaje de las manos y la utilización del mismo guante para la antisepsia e inserción del catéter. A pesar del sitio de video YouTube ser una herramienta muy difundida actualmente, no hay videos que muestren la técnica de acuerdo con lo que se preconiza en la literatura, lo que puede influenciar de modo negativo la población que lo utiliza para fijar el conocimiento.

DESCRIPTORES: Cateterismo urinario; Enfermería; Webcasts; Tecnología educacional. 


\section{INTRODUÇÃO}

O processo de aprendizagem de cursos de graduação em saúde passa por diversas transformações com o objetivo de estabelecer estratégias eficazes para o aprimoramento dos mecanismos de construção de conhecimento dos estudantes $^{(1-2)}$.

Nesse contexto, as novas tecnologias de ensino são alternativas coerentes às transformações no processo de aprendizagem, pois qualificam a formação dos profissionais em saúde, bem como consolidam a interface entre a teoria e prática ${ }^{(1)}$.

Assim, as Tecnologias de Informação e Comunicação ( $\mathrm{TIC}^{\prime}$ 's) são consideradas como ferramentas tecnológicas de aprendizado para formação em enfermagem, permitindo auxiliar, reunir e garantir a melhoria do repasse de informações destinadas aos estudantes ${ }^{(2)}$.

Destaca-se, nesse ínterim, os vídeos enquanto tecnologias educacionais, uma vez que possibilitam orientar atividades práticas, mediante simulação do ambiente de cuidado e da assistência ao paciente. Esses aspectos podem auxiliar os estudantes a sanar as suas principais necessidades e dúvidas, antes de ser inserido em atividade prática real(2). Por essas características, comumente, os vídeos são utilizados por estudantes de nível técnico profissionalizante e de graduação.

Dentre as fontes de consulta de vídeos educativos, destaca-se a Internet, em especial, nas plataformas virtuais como o YouTube e o Google. Contudo, os vídeos indexados nessas plataformas não garantem eficácia e qualidade das informações, demandando uma triagem adequada, pautada em protocolos de ensino.

Desse modo, faz-se necessário analisar a qualidade dos vídeos que são divulgados, pois há a possibilidade de se aprender algo errado e, consequentemente, a execução do procedimento ser incorreta ${ }^{(3)}$.

Destaca-se, nesse estudo, a técnica do cateterismo urinário de demora feminino, procedimento que consiste na introdução de uma sonda de látex ou de plástico no interior da bexiga, chegando até esta através da uretra. O cateter permanente permanece por um maior tempo no paciente, sendo removido quando este é capaz de urinar de modo voluntário( ${ }^{(4)}$.
Uma técnica de cateterização incorreta pode acarretar vários prejuízos ao paciente, entre eles estão infecção urinária, hemorragia, cálculos na bexiga, bexiga neurogênica e trauma tissular ${ }^{(4)}$. Destaca-se, nesse sentido, a importância da atualização constante dos profissionais, bem como de formação acadêmica efetiva.

Assim, diante da constatação de que os vídeos divulgados no Youtube são amplamente utilizados como fonte de pesquisa de estudantes e profissionais de enfermagem ${ }^{(3)}$ e, tendo por base que tais vídeos não possuem um controle de qualidade para que se efetue a sua postagem, torna-se relevante investigar: quais as características dos vídeos compartilhados no Youtube acerca da técnica de cateterismo de demora feminino?

Objetiva-se, destarte, caracterizar os vídeos do Youtube que abordam a técnica de cateterismo urinário de demora feminino.

\section{MÉTODO}

Trata-se de uma pesquisa do tipo descritiva, de abordagem quantitativa. Os dados foram levantados a partir do sítio de compartilhamento de vídeos Youtube, que possui o endereço virtual: www. Youtube.com. Por mais que existam outros sítios de compartilhamentos de vídeos, o Youtube foi escolhido por ser a página mais comum e popular entre os usuários, no momento.

A coleta dos dados ocorreu em 07 de setembro de 2014. Primeiramente, foi utilizado o descritor controlado "cateterismo urinário", extraído dos Descritores em Ciências da Saúde (DeCS), no campo de busca do Youtube. Após, foram aplicados os filtros tipo de resultado e data da postagem disponibilizados pelo próprio sítio, selecionando apenas vídeos cujo upload fosse datado no último ano - 2014.

Para inclusão, os vídeos deveriam seguir os critérios: vídeos que tivessem como objetivo demonstrar a técnica de cateterismo urinário de demora feminino; em linguagem verbal - idioma português, espanhol ou inglês - ou não verbal.

Os critérios de exclusão definidos foram: vídeos que não respondessem à questão de pesquisa e/ou não se referissem à temática; vídeos duplicados; e vídeos acerca do cateterismo urinário de alívio e do cateterismo urinário de 
demora masculino.

Após a seleção, de acordo com os critérios de inclusão, os links dos vídeos foram salvos em uma planilha em um único dia, de modo a não comprometer a amostra selecionada, visto que, a cada acesso, novos vídeos são postados. Desse modo, o conteúdo de cada vídeo foi assistido de forma muito cuidadosa, com o escopo de analisar a técnica do cateterismo de demora.

As visitas ao sítio se deram sem local definido, uma vez que não há restrição de acesso aos vídeos. Dessa forma, foi possível assisti-los várias vezes e extrair as informações necessárias para uma melhor análise, a qual foi efetuada de forma padronizada.

Os indicadores de coleta, com suas respectivas formas de análise, foram: 1) tempo de duração: indicado na timeline do vídeo, em minutos e segundos; 2) responsável pela postagem: pessoa física, blogs, empresas; 3) data da postagem; 4) total de visualizações; 5) categoria: segundo classificação do Youtube; 6) tipo de procedimento: masculino, feminino ou ambos; e 7) execução da técnica: correta ou incorreta. Tais indicadores foram desenvolvidos tomando por base trabalhos com metodologia semelhante, que também buscaram analisar vídeos do Youtube ${ }^{(3)}$.

Para definir se a execução da técnica foi correta ou incorreta, analisou-se a adequação das etapas componentes do procedimento (Quadro 1), que foram definidas a partir de uma revisão literária, realizada em junho de 2014 nas bases de dados Scientific Eletronic Library Online (SciELO), Literatura Latino-Americana em Ciências da Saúde (LILACS), PubMed, Cumulative Index to Nursing and Allied Health Literature (CINAHL), Scopus e Cochrane.

A análise dos dados se deu a partir da estatística descritiva simples (frequência absoluta e relativa). Como a pesquisa não está envolvida de forma direta com seres humanos, não houve a necessidade da aprovação em comitê de ética.

Quadro 1 - Etapas componentes da técnica de cateterismo urinário de demora feminino. Natal-RN-Brasil, 2014

\section{Etapa}

a) Prescrição do cateterismo urinário de demora, com verificação da indicação correta ${ }^{(5-6)}$.

b) Planejamento do procedimento, com organização do material necessário para a inserção do cateter asséptico: dois pares de luvas estéreis; gaze estéril; iodo-séptico ou sabonete de clorexidina; sacos para resíduos; cateter uretral de acordo com o paciente e a indicação do cateterismo; seringa com 7 ou $8 \mathrm{ml}$ de água destilada; anestésico lubrificante hidrossolúvel; seringa 3 ou $4 \mathrm{ml}$ (opcional); recipiente para urina; equipo fechado para drenagem; campo estéril (opcional); fixação de tiras adesivas (opcional) $)^{(7-8)}$.

c) Realização da técnica ${ }^{(7-9)}$

1. Reúna o material necessário para a inserção do cateter asséptico; 2. Lave as mãos; 3 Antissepsia: em um lugar onde a privacidade é garantida, coloque a paciente em decúbito dorsal, com as pernas afastadas e os joelhos dobrados; coloque as luvas, com o dedo indicador e o polegar da mão não dominante separe cuidadosamente os lábios e exponha o meato, lave com sabonete antisséptico, nesta ordem: grandes lábios, pequenos lábios, clítoris, meato, e siga para o períneo, com movimentos de cima para baixo sem retornar; seque na mesma direção e repita o procedimento duas vezes; 4 . Mude de luvas; 5 . Coloque o campo estéril; 6 . Pegue a sonda e lubrifique a ponta, enrole-a dentro de sua mão dominante para evitar contaminá-la. Com o polegar e o indicador da outra mão separe os grandes lábios, localize o meato uretral, e se não for observado, como em algumas mulheres obesas ou multíparas, palpe-o; quando tiver certeza da sua localização inserir o cateter delicadamente, mas firme; quando a urina começa a fluir avançar a sonda $10 \mathrm{~cm} ; 7$. Verifique a localização do cateter na bexiga - pela presença de urina - e infle o balão com 5 ou $7 \mathrm{ml}$ de água destilada ou estéril, realize a tração para a sonda, suavemente, ancorar no colo da bexiga e remova o campo estéril com cuidado para não contaminar a porção distal da sonda; 8. Prenda a sonda no sistema de drenagem fechado; 9. Prenda a sonda na parte inferior do abdome para evitar lesões da uretra pela tração exercida; 10. Limpe e seque a paciente, cubra e deixe confortável.

d) Registrar o procedimento no prontuário da paciente ${ }^{(6)}$.

e) Monitorar e avaliar continuamente o cateter uretral, mantendo a comunicação e a cooperação com a paciente ${ }^{(7-9)}$.

\section{RESULTADOS}

A busca de vídeos no sítio do Youtube totalizou um quantitativo de 8.280 vídeos, os quais, a partir da aplicação dos filtros, resultaram em 499 (6,0\%) arquivos pré-selecionados; estes, por sua vez, foram analisados individualmente, com base nos critérios de inclusão e de exclusão e nos indicadores de coleta de dados, totalizando uma amostra de 29 vídeos, valor correspondente 
a $0,35 \%$ do valor encontrado inicialmente.

A caracterização dos vídeos que compõem a amostra do estudo está representada na Tabela 1, sendo possível observar os indicadores: tempo de duração, autor, data da postagem, categoria e execução da técnica.

Constatou-se uma predominância de vídeos com duração intermediária, de 4 a 20 minutos (23; 79,4\%); ao todo, os 29 vídeos totalizaram 4h47min59s de vídeo assistido, uma média de 10min19s por vídeo.

A maioria deles foi postada por pessoa física (24; $82,8 \%)$, tendo em vista que se trata de um sítio de compartilhamento de vídeos aberto, em que qualquer pessoa pode ter acesso.

Quanto à categoria dos vídeos, determinada pelo próprio publicador e demonstrada na página do vídeo no Youtube, houve uma predominância da categoria pessoas e blogs (18; 62\%), o que não condiz com o observado no conteúdo dos vídeos, já que a maioria tinha um cunho educativo.

Através da avaliação da técnica de cateterismo urinário de demora feminino demonstrada no vídeo, tendo por base a sequência descrita na literatura (Quadro 1), evidenciou-se uma prevalência de procedimentos incorretos (26; 89,7\%). As principais incoerências estão destacadas na Figura 1.

Destaca-se, então, como principais erros encontrados nos vídeos analisados, a ausência de registro do procedimento no prontuário (25; $86,2 \%)$, não demonstração da lavagem das mãos (23; 79,4\%) e a utilização da mesma luva para a antissepsia e inserção da sonda (18; 62\%).
Tabela 1 - Caracterização dos vídeos integrantes da amostra do estudo. Natal-RN-Brasil, 2014

\begin{tabular}{lcc}
\hline Indicador de análise & $\mathbf{n}$ & $\mathbf{\%}$ \\
\hline Tempo de duração & & \\
\hline Curto (menos de 4') & 3 & 10,3 \\
\hline Intermediário (entre 4' e 20') & 23 & 79,4 \\
\hline Longos (mais de 20') & 3 & 10,3 \\
\hline Autor & & \\
\hline Pessoa física & 24 & 82,8 \\
\hline Órgão & 0 & 0 \\
\hline Empresa & 5 & 17,2 \\
\hline Data da postagem & & \\
\hline 2013 & 17 & 58,6 \\
\hline 2014 & 12 & 41,4 \\
\hline Categoria & & \\
\hline Pessoas e blogs & 18 & 62 \\
\hline Educação & 5 & 17,2 \\
\hline Ciência e tecnologia & 2 & 6,8 \\
\hline Notícias e política & 1 & 3,5 \\
\hline Entretenimento & 1 & 3,5 \\
\hline Filmes e desenhos & 1 & 3,5 \\
\hline Comédia & 1 & 3,5 \\
\hline Execução da técnica & & \\
\hline Correta & 3 & 10,3 \\
\hline Incorreta & 26 & 89,7 \\
\hline & & \\
\hline & & \\
\hline & & \\
\hline
\end{tabular}

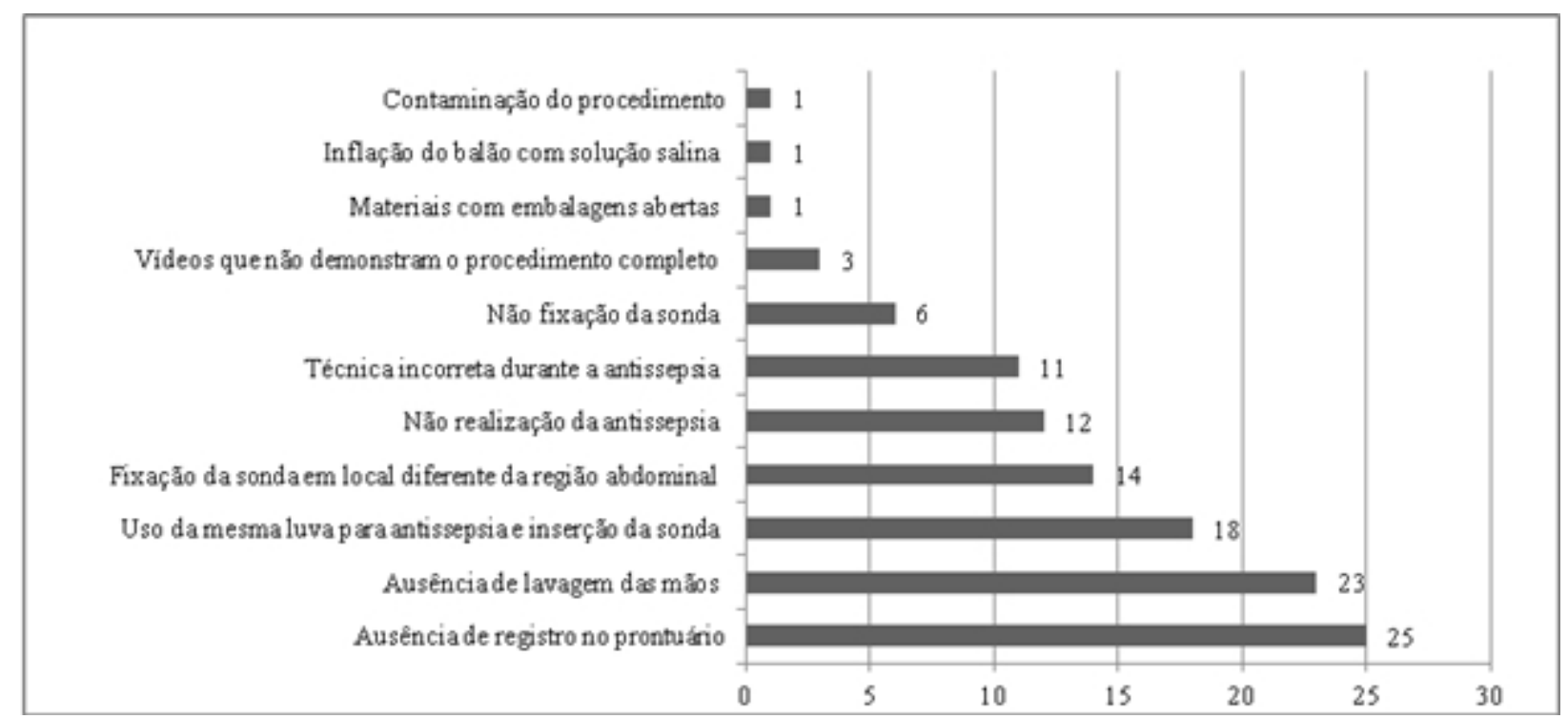

Figura 1 - Principais erros observados nos vídeos analisados acerca do cateterismo urinário de demora feminino, em número absoluto. Natal-RN-Brasil, 2014 


\section{DISCUSSÃO}

Com o advento da Internet, a propagação da informação se tornou cada vez mais veloz, e, por consequência, muitos recursos têm sido agregados ao cotidiano das pessoas ${ }^{(10)}$.

O sítio do Youtube, desse modo, ganha destaque na contemporaneidade e constitui uma importante ferramenta de divulgação de informação por meio de recursos audiovisuais, através da publicação e compartilhamento de vídeos ${ }^{(10)}$.

Dentre suas inúmeras funções, realça-se a possibilidade de postagem e utilização dos vídeos de maneira educativa, aspecto que põe em relevo a necessidade de se analisar a qualidade dos conteúdos divulgados. Assim, a confiabilidade desses dados transmitidos merece atenção especial, principalmente, quando se trata da saúde.

Dos 29 vídeos analisados que abordam a técnica do cateterismo urinário de demora feminino, 26 não estavam de acordo com o preconizado pela literatura, evidenciando a publicação demasiada de vídeos sem o comprometimento com a informação que está sendo transmitida.

De forma semelhante, um estudo ${ }^{(3)}$ que analisou os vídeos quanto à técnica de ressuscitação cardiopulmonar $(\mathrm{RCP})$, verificou que a maioria estava desatualizado, de acordo com as antigas diretrizes de RCP. Apreende-se, desse modo, a veiculação de conteúdo que contradiz os preceitos estabelecidos pela literatura e, com isso, as pessoas que utilizam esse recurso no intuito educativo precisam ter discernimento quanto ao conteúdo que estão assistindo, caso contrário, receberão uma influência negativa, podendo propagar essa informação errônea em seu cotidiano.

Vale destacar que não se trata de uma realidade unânime: outro trabalho ${ }^{(11)}$, ao analisar os vídeos do Youtube sobre aleitamento materno, constatou que os mesmos faziam referência aos fatores relevantes dessa prática, exercendo influência positiva aos visualizadores.

Desse modo, realça-se que vídeos que veiculem informação adequada podem se traduzir em importante material de apoio ao ensino das técnicas de enfermagem, em consonância com os comprovados benefícios de utilização das TIC's como ferramenta de ensino, em especial, as hipermídias - união de diversas mídias, como texto, vídeos, hipertextos, gravuras, animações ${ }^{(12-14)}$.

Dentre os erros encontrados nos vídeos analisados, a ausência dos registros de enfermagem merece destaque. Sabe-se que as informações são centrais no processo de cuidar em saúde. Por meio do registro do processo de cuidar é que se pode assegurar a execução e a continuidade da atenção em saúde, bem como viabilizar para a equipe de saúde a prestação de atendimento respaldado no conhecimento e nos aspectos éticos e legais ${ }^{(15)}$.

As informações em saúde, destarte, constituem a base para a comunicação dos profissionais de saúde, elemento basilar para a garantia da segurança do paciente e para a qualificação do cuidado prestado.

Estima-se que $50 \%$ das informações referentes ao cuidado são fornecidas pela enfermagem ${ }^{(16)}$. Diante de uma cifra significativa, espera-se que os registros realizados por esta categoria permitam a comunicação permanente entre os membros da equipe multiprofissional, com transmissão de informações que facilitem o planejamento, tomada de decisões clínicas e gerenciais e continuidade da assistência prestada.

Os registros de enfermagem, por conseguinte, são considerados como um dos meios de demonstrar o trabalho executado pela equipe de enfermagem e um indicador de qualidade da assistência ${ }^{(17)}$, sendo imprescindível que sejam veiculados como etapa componente de todos os procedimentos de enfermagem, inclusive o cateterismo urinário de demora feminino.

Outro ponto observado foi a ausência de demonstração da lavagem das mãos em 23 vídeos, aspecto que comprovadamente aumenta o risco de ocasionar infecção do trato urinário (ITU) durante a realização do procedimento e a possibilidade de contaminação cruzada ${ }^{(5)}$.

Vale salientar que a higienização das mãos é uma medida simples e rápida de prevenir a proliferação da infecção relacionada à assistência em saúde, devendo ser realizada por todos os profissionais e familiares, e, dentre outras situações, antes e após o contato com o paciente $^{(18)}$.

Ainda em relação aos erros observados, destaca-se a utilização da mesma luva para a realização da antissepsia e inserção da sonda, 
trazendo, também, um risco adicional de ITU.

Ao analisar as etapas dos procedimentos descritas, mostraram-se ser recorrentes nos vídeos: verificação da posição do cateter e inflação do balão; lubrificação e inserção do cateter; conexão do sistema de drenagem fechado à sonda; colocação do campo estéril; e planejamento do procedimento.

Ressalta-se que, ao se certificar da correta inserção do cateter através do refluxo da urina e posterior inflação do balão, garante-se ao paciente um correto posicionamento da sonda na bexiga, aspecto importante para a prevenção de danos ao paciente ${ }^{(5,19)}$.

Destaca-se, ainda, que o balão deve ser preenchido com água destilada, pois as soluções salinas e com outros eletrólitos trazem risco de cristalização, dificultando a sua deflação no momento de retirada do cateter $^{(19)}$. Um vídeo analisado demonstrou incorretamente o preenchimento do balão com solução salina.

No que concerne à lubrificação e inserção do cateter de forma delicada, apreende-se que tais cuidados oferecem ao paciente um menor risco de trauma da uretra, facilitando a sua inserção, bem como aliviando a dor causada pelo bexigoma $^{(19)}$.

Da mesma forma, quando se conecta o sistema de drenagem fechado à sonda, evitando que haja a sua desconexão, reduz-se o risco de contaminação. Tais sistemas devem conter dispositivos que visem à minimização de infecção, como: válvula antirrefluxo, câmera de gotejamento e local para coleta de urina ${ }^{(20)}$.

Por fim, destaca-se a importância da etapa de planejamento do procedimento, a qual é indispensável para a garantia de uma técnica asséptica, evitando que as etapas sejam quebradas em virtude da falta ou inadequação de material|(20-21), além da preocupação de garantia de um cuidado integral, atentando para a indicação correta do uso do cateter de demora, aspecto que deve ser compreendido a partir da equipe multiprofissional de saúde.

\section{CONCLUSÃO}

A partir do estudo, verificou-se uma quantidade significativa de vídeos que abordam a técnica do cateterismo urinário de demora feminino incompatíveis com a técnica padronizada pela literatura, tendo potencial de influenciar negativamente o profissional ou acadêmico de enfermagem que os assistem, comprometendo, assim, a assistência prestada ao paciente.

O sítio do Youtube constitui-se uma tecnologia que pode subsidiar o processo de ensinoaprendizagem, no entanto, as pessoas que o buscam com o intuito educativo precisam ter um conhecimento teórico prévio para reconhecer os erros presentes nos conteúdos publicados, principalmente quando se trata da saúde, a fim de não propagar a informação equivocada.

Devido a essa carência e considerando-se o grande acesso a esse sítio de informação, vislumbra-se a necessidade de publicações de vídeos relacionados à temática em questão que estejam de acordo com o preconizado pela literatura, a fim de proporcionar aos profissionais e acadêmicos de enfermagem fonte confiável em relação à técnica demonstrada.

\section{REFERÊNCIAS}

1. Bastable SB. O enfermeiro como educador. $3^{\mathrm{a}}$ ed. São Paulo: Artmed; 2010.

2. Boctor L. Active-learning strategies: the use of a game to reinforce learning in nursing education. A case study. Nurse education in practice. 2013;13(2):96-100.

3. Tourinho FSV, Medeiros KS, Salvador PTCO, Castro GLT, Santos VEP. Análise de vídeos do Youtube sobre suporte básico de vida e reanimação cardiopulmonar. Rev. Col. Bras. Cir. 2012;39(4):335-9.

4. Potter PA, Perry AG. Fundamentos de enfermagem. $7^{\mathrm{a}}$ ed. Rio de Janeiro: Elsevier; 2009.

5. Jansen IAV, Hopmans TEM, Wille JC, Van den Broek PJ, Van der Kooi T, Van Benthem BHB. Appropriate use of indwelling urethra catheters in hospitalized patients: results of a multicentre prevalence study. BMC Urol. [Internet] 2012;12(25) [acesso em 04 jan 2015]. Disponível: http://www.ncbi.nlm.nih.gov/pmc/ articles/PMC3502298/pdf/1471-2490-12-25.pdf.

6. Conterno LO, Lobo JA, Masson W. Uso excessivo do cateter vesical em pacientes internados em enfermarias de hospital universitário. Rev. Esc. Enferm. USP. 2011;45(5):1089-96.

7. Todsen $\mathrm{T}$, Henriksen MV, Kromann CB, Konge L, Eldrup J, Ringsted C. Short- and long-term transfer of urethral catheterization skills from simulation training to performance on patients. BMC Med Educ. [Internet] 
2013;13(29) [acesso em 04 jan 2015]. Disponível: http:// www.biomedcentral.com/content/pdf/1472-6920-1329.pdf.

8. Arango AMA, Maya MPV, Pérez DMP, Zuluaga JIM, Monsalve LMM, Zapata CPM. Caracterización clínica y epidemiológica de los pacientes con infección del tracto urinario asociadas al cuidado de la salud. Invest. Educ. Enferm. [Internet] 2012;30(1) [acesso em 12 mar 2015]. Disponível: http://www.scielo.org.co/pdf/iee/ v30n1/v30n1a04.pdf.

9. Al Mohajer M, Darouiche RO. Prevention and treatment of urinary catheter-associated infections. Curr. Infect. Dis. Rep. 2013;15(2):116-23.

10. Pellegrini DP, Reis DD, Monção PC, Oliveira R. Youtube: Uma nova fonte de discursos. [Internet] 2013 [acesso em 20 jan 2015]. Disponível: http://www.bocc. ubi.pt/pag/bocc-pelegrini-cibercultura.pdf.

11. Carvalho JA, Gurgel PKF, Lima KYN, Dantas CN, Martins CCF. Análise de vídeos do Youtube sobre aleitamento materno: importância e benefício. Rev. enferm. UFPE on line. 2013;7(Esp):1016-22.

12. Freitas LV. Construção e validação de hipermídia educacional em exame físico no pré-natal [dissertação]. Fortaleza (CE): Universidade Federal do Ceará; 2010.

13. Onda EL. Situated cognition: its relationship to simulation in nursing education. Clinical Simulation in Nursing. 2012;8(7):273-80.

14. Nickerson CJ, Thurkettle MA. Cognitive Maturity and Readiness for Evidence-Based Nursing Practice. J. Nurs. Educ. 2013;52(1):17-23.

15. Sousa PAF, Dal Sasso GTM, Barra DCC. Contribuições dos registros eletrônicos para a segurança do paciente em terapia intensiva: uma revisão integrativa. Texto Contexto Enferm. 2012;21(4):971-9.

16. Silva JA, Grossi ACM, Haddad MCL, Marcon SS. Avaliação da qualidade das anotações de enfermagem em unidade semi-intensiva. Esc. Anna Nery. 2012;16(3):576-82.

17. Franco MTG, Akemi EN, D'inocento M. Avaliação dos registros de enfermeiros em prontuários de pacientes internados em unidade de clínica médica. Acta Paul. Enferm. 2012;25(2):163-70.

18. Ministério da Saúde (BR). Agência Nacional de Vigilância Sanitária (ANVISA). Segurança do paciente: higienização das mãos. [Internet] 2012 [acesso em 20 jan 2015]. Disponível: http://www.anvisa.gov.br/ servicosaude/manuais/paciente_hig_maos.pdf.

19. Smeltzer SC, Bare BG, Hinkle JL, Cheever KH. Tratado de enfermagem médico-cirúrgica. $11^{\mathrm{a}} \mathrm{ed}$. Rio de Janeiro: Guanabara Koogan; 2009.
20. Campos CVS, Silva KL. Cateterismo urinário intermitente realizado pelos cuidadores domiciliares em um serviço de atenção domiciliar. REME, Rev. Min. Enferm. 2013;17(4):753-62.

21. Mendonça AEO, Dantas JG, Andrade DA, Segato CT, Torres GV. Perfil sociodemográfico e clínico de idosos submetidos à hemodiálise. Cogitare enferm. [Internet] 2015;20(1) [acesso em 10 abr 2015]. Disponível: http://ojs.c3sl.ufpr.br/ojs2/index.php/cogitare/article/ view/37080/24841. 